Research Paper

\title{
Left Stellate Ganglion Ablation Inhibits Ventricular Arrhythmias through Macrophage Regulation in Canines with Acute Ischemic Stroke
}

\author{
Youcheng Wang, $\mathrm{MD}, \mathrm{PhD}^{* 1}$, Shanqing He, MD, PhD ${ }^{*}$, Xiaoxing Xiong, MD, PhD², Jia Liu, MD, PhD2, \\ Baojun Xie, MD, PhD², Yajun Yao, MD, PhD1, Junkui Yin, MD¹, Liuliu Zi, MD¹, Xi Wang, MD, PhD1, \\ Yanhong Tang, MD, $\mathrm{PhD}^{1}$, Qingyan Zhao, MD, $\mathrm{PhD}^{1 凶}$ \\ 1. Department of Cardiology, Renmin Hospital of Wuhan University, Cardiovascular Research Institute of Wuhan University, Hubei Key Laboratory of \\ Cardiology, Wuhan City, Hubei Province, China \\ 2. Department of Neurosurgery, Renmin Hospital of Wuhan University, Wuhan City, Hubei Province, China \\ 3. Department of Radiology, Renmin Hospital of Wuhan University, Wuhan City, Hubei Province, China \\ *Drs Wang and He are the co-first authors.
}

$\square$ Corresponding author: Qingyan Zhao, Cardiovascular Research Institute of Wuhan University, Renmin Hospital of Wuhan University, 238 Jiefang Road, Wuhan, China. Email: ruyan71@163.com

(1) The author(s). This is an open access article distributed under the terms of the Creative Commons Attribution License (https://creativecommons.org/licenses/by/4.0/). See http://ivyspring.com/terms for full terms and conditions.

Received: 2020.07.22; Accepted: 2020.12.18; Published: 2021.01.01

\begin{abstract}
Aims: To investigate the potential mechanism of ventricular arrhythmias (VAs) after acute ischemic stroke and explore the effects of left stellate gangling (LSG) ablation on VAs induced by stroke in canines.

Materials and Methods: Twenty canines were randomly divided into the sham-operated group $(n=6)$, AS group $(n=7)$ and SGA group $(n=7)$. Cerebral ischemic model was established in the AS group and the SGA group by right acute middle cerebral artery occlusion (MCAO). LSG ablation was performed in the SGA group as soon as MCAO. After 3 days, atrial electrophysiology and neural activity were measured in vivo. The levels of norepinephrine (NE) in plasma and ventricle were detected by ELISA. The levels of monocyte chemotactic protein-1 (MCP-1), tumor necrosis factor- $\alpha$ (TNF- $\alpha$ ) and NF-KB p65 in ventricle were detected by western blotting. The pro-inflammatory polarization of macrophages in ventricle was detected by immunofluorescence.

Results: Higher ventricular tachycardia (VT) inducibility and lower ventricular fibrillation threshold (VFT) were observed in the AS group compared with those in the sham-operated group, associated with higher LSG activity and NE levels, increased number of MI macrophages and secretion of inflammatory cytokines in ventricle (all $\mathrm{P}<0.001$ ). Compared with the AS group, the SGA group had lower VT inducibility and higher VFT, combined with lower NE levels, and reduced number of $M 1$ macrophages and secretion of inflammatory cytokines in ventricle (all $\mathrm{P}<0.001$ ).

Conclusion: LSG ablation could reduce VAs vulnerability after acute stroke by preventing the macrophages polarization and activation induced by sympathetic hyperactivity.
\end{abstract}

Key words: acute stroke; ventricular arrhythmia; sympathetic nerve; macrophage; canine

\section{Introduction}

The effects of cerebrovascular diseases on the modulation of autonomic nervous system and cardiovascular function have been studied for decades. Early observations showed that electrocardiographic (ECG) abnormalities and cardiac arrhythmias occurred in almost half of patients with an acute stroke [1]. Although most patients died directly from the neurological injury during the first week after the stroke, about 2 to $6 \%$ of all stroke patients died from cardiac causes in the first 3 months and approximately $19 \%$ had fatal or serious non-fatal cardiac events [2]. An increased risk of ventricular 
arrhythmias (VAs) is one of the most serious cardiac outcomes after stroke, leading to a high risk of sudden death [3]. However, the exact mechanism of VAs and sudden death after acute stroke remains incompletely understood.

Previous studies have shown that acute stroke is often accompanied by autonomic dysfunction, especially sympathetic excessive activity, generally manifested as the increased blood pressure, a higher low frequency/high frequency ratio of heart rate variability, or the increased norepinephrine (NE) and cortisol levels in blood [4-6]. It has been confirmed that the sympathetic excessive activity and the sympathetic nerve sprouting in myocardium are important factors leading to the occurrence of VAs in acute myocardial infarction [7]. As is known to all, stellate ganglion (SG), which belongs to sympathetic ganglion in function, is an important pathway connecting sympathetic nerve and cardiac autonomic system. NE, the major neurotransmitter released from postganglionic sympathetic nerve terminals, can directly bind to $\beta$-adrenergic receptors on myocardial cell membrane, resulting in effective refractory period (ERP) and action potentials shortening, and cardiac automaticity increasing. Meanwhile, NE is also known to be a potential pro-inflammatory mediator and can induce the release of multiple inflammatory factors $[8,9]$. Previous studies have suggested that methods inhibiting the sympathetic activity, such as stellate ganglion ablation (SGA) and renal sympathetic denervation, have an positive effect on the myocardial electrophysiological changes and can prevent the occurrence of VAs during acute myocardial infarction [10, 11]. In this study, we investigated the potential mechanism of VAs after acute ischemic stroke and explored the effects of SGA on VAs induced by stroke in canines.

\section{Materials and methods}

This study was approved by the animal studies subcommittee of our institutional review board and was in compliance with the guidelines of the National Institutes of Health for the care and use of laboratory animals.

\section{Animal model preparation}

An intramuscular injection of $25 \mathrm{mg} / \mathrm{kg}$ ketamine sulfate was administered before pentobarbital sodium (ASPEN Biotechnology Co., Ltd, China) premedication. All of the canines were premedicated with sodium pentobarbital $(30 \mathrm{mg} / \mathrm{kg}$, IV), intubated, and ventilated with room air supplemented with oxygen from a respirator (MAO01746, Harvard Apparatus Holliston, USA).
Continuous ECG monitoring was performed. Twenty beagle canines (weighing 9 to $10 \mathrm{~kg}$ ) were randomly assigned to 3 groups. The sham-operated group consisted of 6 canines that underwent craniotomy without right middle cerebral artery occlusion (MCAO). The AS group consisted of 7 canines in which a cerebral ischemic model was established through the occlusion of the right middle cerebral artery. The SGA group consisted of 7 canines that underwent the left stellate ganglion (LSG) ablation as soon as MCAO was completed.

All the operations followed the principle of sterility, and prophylactic antibiotics were given in the perioperative period.

\section{MCAO}

To establish the model of acute ischemic stroke, MCAO on canines was performed. After successful anesthesia, right craniotomy was performed to expose the trunk of the right middle cerebral artery under a microscope. The trunk was stanched by bipolar electrocoagulation and then cut off. Detailed operational procedures were shown in the previous study [12]. Magnetic resonance imaging (MRI) of the head was performed 24 hours after the operation to ensure the success of the stroke model. The MRI images are shown in Figure 1.

\section{LSG ablation}

The LSG was completely exposed by left thoracotomy through the second intercostal space. A radiofrequency current (30-35 W, $150 \mathrm{~s}$ ) was delivered to the site by an electrode catheter (Biosense Webster, Inc, Diamond Bar, CA) showing blood pressure (BP) elevation during stimulation. Complete ablation was verified by the abolishment of BP elevation during the delivery of electrical stimulation to the ablated site.

\section{Recording of LSG activity}

LSG activity was measured in the sham-operated group and the AS group 3 days after the operations. To avoid the disturbance of electrical stimulation, the recordings of autonomic neural activity were performed before the electrophysiological measurements. Pairs of bipolar hook electrodes (Xi'an Friendship Medical Electronics Co., Ltd., China) were attached to the LSG. All measured signals were amplified and filtered between $0.3 \mathrm{~Hz}$ and $1 \mathrm{kHz}$ with a PowerLab system (AD Instruments, Dunedin, New Zealand). Neural signals that were three times higher than noise signal were marked. Neural activity was represented by the frequency (per minute) and amplitude of neural discharges. 

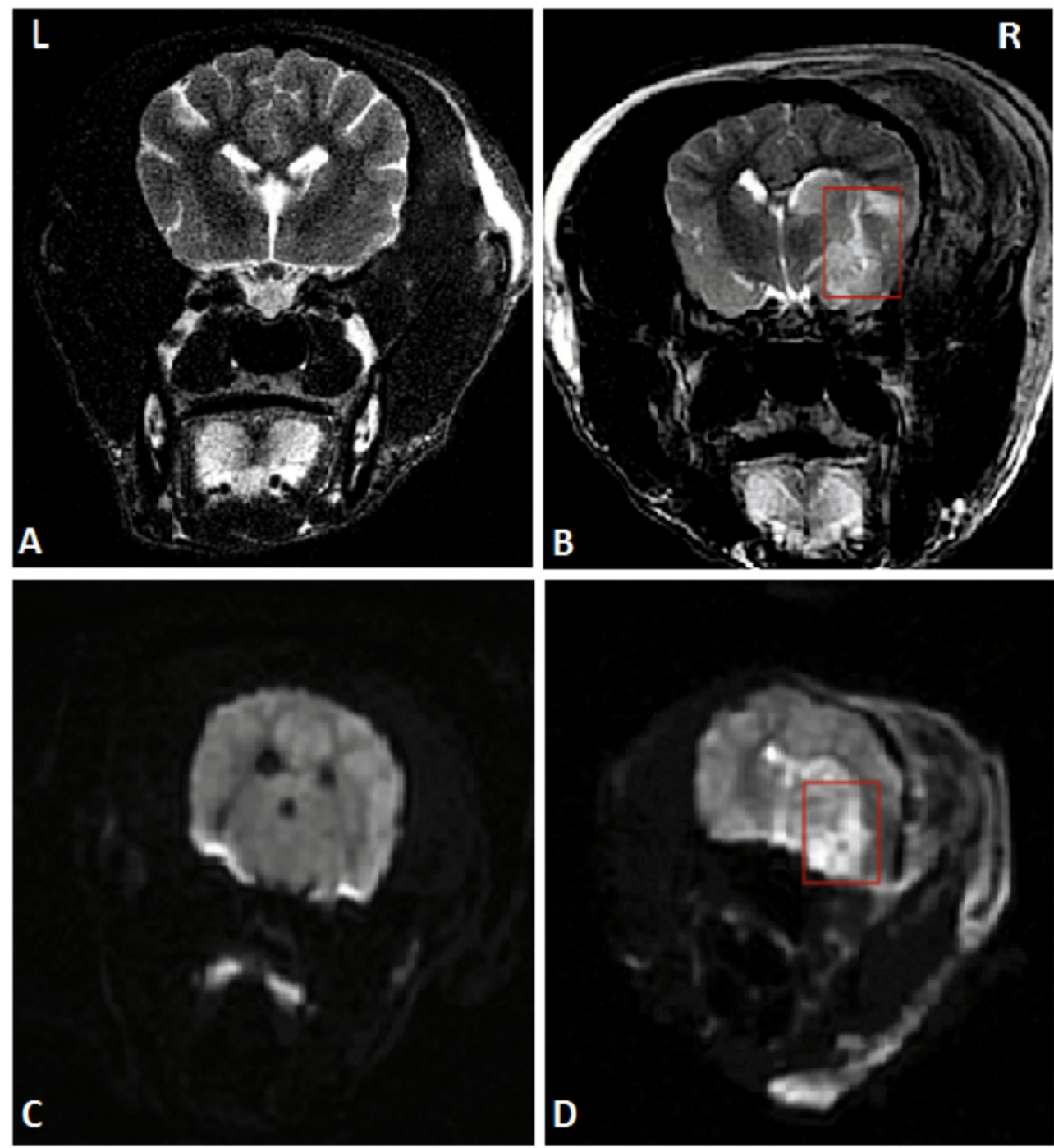

Figure 1: A head MRI that was performed 24 hours after MCAO suggested the right temporal lobe infarction, marked by red rectangles. (A) and (C) Coronal MRI scan of the sham-operated group in T2WI and DWI respectively. (B) and (D) Coronal MRI scan of the AS group in T2WI and DWI respectively. Abbreviations: AS: acute stroke; SGA: stellate ganglion ablation; MCAO: middle cerebral artery occlusion; MRI: magnetic resonance imaging; T2WI: T2 weighted imaging; DWI: diffusion weighted imaging.

\section{Electrophysiological measurements}

The stimuli were delivered with a computerized electrophysiology system (Lead 7000, Jinjiang Inc., China). ERPs in the right ventricular epicardium at the apex (RVA), free wall (RVFW), base (RVB) and left ventricular epicardium at the apex (LVA), free wall (LVFW), base (LVB) were determined during ventricular pacing at $300 \mathrm{~ms}$ cycle length using stimuli at twice threshold. A single premature stimulus (S2) was introduced after 8 basic stimuli (S1), starting with a coupling interval of $250 \mathrm{~ms}$ and reducing by $10 \mathrm{~ms}$ decrement. As the S1-S2 intervals approached the ERP, decrement was reduced to $2 \mathrm{~ms}$. ERP was the longest S1-S2 interval at which S2 failed to capture. ERP dispersion (dERP) was the maximum difference among all sites tested.

ECG was continuously recorded for 1 hour to calculate the number of spontaneous VAs. VAs were classified as ventricular premature beat (VPB), ventricular tachycardia (VT; $\geq 3$ consecutive ventricular premature beats), and ventricular fibrillation (VF).

Inducibility of VAs was assessed with programmed ventricular stimulation from right free wall. Single (S2) and double (S3) extrastimuli were delivered after 8 beats of ventricular drive (S1) at 300 ms (twice threshold, 2 ms duration). Stimulation was terminated when VT or VF was induced.

The ventricular fibrillation threshold (VFT) was measured at the end of the study. VF was evoked by programmed electrical stimulation at the right ventricular epicardium (a fixed train of 30 stimuli, $30 \mathrm{~ms}$ interval). The VFT was determined by the progressively increasing pacing voltage from $1 \mathrm{~V}$, with a $30 \mathrm{~s}$ rest period before the next pacing train if no VF was induced. VF was defined as chaotic, fractionated electrical activity persisting for $>5 \mathrm{~s}$ and 
then was electrically defibrillated to the sinus rhythm.

\section{Enzyme-linked immunosorbent assay (ELISA)}

Blood samples from jugular vein separately obtained at baseline and 3 days after operations and tissue specimens that were obtained from the left ventricle $(\mathrm{LV})$ were temporarily stored at $-80^{\circ} \mathrm{C}$ until the assay. Then, samples from all groups were assigned to measure NE levels examined by General NE ELISA Kit (ELK Biotechnology, China), based on the protocol provided by the manufacturer.

\section{Immunofluorescence}

At the end of measurements, the ventricular tissues were quickly removed and fixed in $4 \%$ paraformaldehyde, embedded in paraffin and sectioned at 5 microns. To identify the pro-inflammatory M1 macrophages, the ventricular samples were incubated with primary antibodies against CD68 (1:100, Abcam, Inc., UK) and inducible nitric oxide synthase (iNOS; 1:50, Abcam, Inc., UK), and cells were stained with DAPI for nuclei. The results were analyzed by Image-Pro Plus 6.0 software. More than 3 sections of ventricle from different samples were randomly selected to quantify the number of macrophages.

\section{Real-time PCR}

The expression of iNOS in ventricle was measured by real-time PCR. Total RNA was isolated from ventricular samples with Tripure Extraction Reagent (ELK Biotechnology, China) according to the manufacturer's protocol. cDNA was synthesized using EntiLink ${ }^{\mathrm{TM}}$ 1st Strand cDNA Synthesis Kit (ELK Biotechnology, China). Real-time fluorescent quantitative PCR was performed on the StepOne real-time PCR instrument (Life technologies, Gaithersburg, MD), and each sample was made into three duplications using EnTurbo ${ }^{\mathrm{TM}}$ SYBR Green PCR SuperMix kit (ELK Biotechnology, China). Primers used for RT-PCR were: Dog-iNOS: 5'-ACCAAT ACAGGCTCGTGCAG-3'(forward),5'-GGGCTGTCT ACTACTCGCTCC-3' (reverse);Dog-GAPDH:5'-GAA GGTCGGAGTGAACGGATT-3'(forward),5'-CATTTG ATGTTGGCGGGATC-3'(reverse).

\section{Western blotting}

The membranes were incubated with a primary antibody against monocyte chemotactic protein-1 (MCP-1; Abcam, Inc., UK), tumour necrosis factor-alpha (TNF- $\mathrm{a}$; Abcam, Inc., UK), NF-kB p65 (Abcam, Inc., UK). The membranes were blocked with $5 \%$ nonfat dry milk in Tris-buffered saline with Tween 20 (TBST) for 1 hour and incubated with the primary antibody overnight at $4{ }^{\circ} \mathrm{C}$. They were then washed in TBST three times, incubated with the secondary antibody for 1 hour at $37{ }^{\circ} \mathrm{C}$, and imaged using Immun-Star horseradish peroxidase substrate. The relative expression levels of the proteins were determined using image analyzer software (AlphaEase FC, San Leandro, CA, USA).

\section{Statistical analysis}

The data were expressed as the mean \pm standard deviation. Two-sample independent Student's t-tests were used to compare the means of two groups. ANOVA followed by Newman-Keuls tests was used to compare the mean values of continuous variables among multiple groups, and any significant differences were further analyzed using the Tukey-Kramer test. All the statistical tests were two-sided, and a probability value $<0.05$ was required for statistical significance.

\section{Results}

\section{Measurements of ventricular ERPs and dERP}

As shown in Figure 2A, the ERPs at all recording sites in the AS group were significantly reduced than those in the sham-operated group and the SGA group (all $\mathrm{P}<0.05)$. For example, the ERP at RVB was $179 \pm 11$ $m s$ in the AS group, while the ERP at RVB was $208 \pm 9$ $\mathrm{ms}$ in the sham-operated group and $193 \pm 10 \mathrm{~ms}$ in the SGA group. Compared with those in the sham-operated group, the ERPs at RVB, LVB and LVFW were lower in the SGA group $(\mathrm{P}<0.05)$. However, there were no significant differences in ERPs at RVFW, RVA and LVA between the sham-operated group and the SGA group.

As shown in Figure 2B, the dERP in the AS group was significantly increased than that in the sham-operated group $(25 \pm 3$ versus $15 \pm 4 \mathrm{~ms}$, $\mathrm{P}<0.001)$ and the SGA group $(25 \pm 3$ versus $19 \pm 3 \mathrm{~ms}$, $\mathrm{P}<0.01)$. The dERP was higher in the SGA group $(\mathrm{P}<0.05)$ than that in the sham-operated group.

\section{Recording of Spontaneous VAs}

During the ECG monitoring, no spontaneous VF was observed in all groups. Only one canine in the AS group had one short episode of VT. The number of spontaneous VPB in the AS group was significantly increased than that in the sham-operated group (34 \pm 13 versus $4.5 \pm 6, P<0.01)$ and the SGA group $(34 \pm 13$ versus $14 \pm 10, \mathrm{P}<0.01)$. Compared with the sham-operated group, an increasing trend of number of VPB in the SGA group was observed, but there was no statistical significance (Figure 3B).

\section{Measurements of VAs inducibility and VFT}

As shown in Figure 3C, the programmed ventricular stimulation induced one short episode of VT in only one canine in the sham-operated group. In 
the AS group, the short episode of VT was induced in four canines, and sustained VT was induced in two canines. In the SGA group, the short episode of VT was induced in two canines. There was no VF induced in all the groups. As shown in Figure 3D, VFT in the AS group was significantly reduced than that in the AS group ( $3.0 \pm 0.8$ versus $9.0 \pm 1.4 \mathrm{~V}, \mathrm{P}<0.001)$ and the SGA group $(3.0 \pm 0.8$ versus $7.1 \pm 1.3 \mathrm{~V}, \mathrm{P}<0.001)$. Compared with that in the sham-operated group, the VFT was lower in the SGA group $(\mathrm{P}<0.05)$.

\section{Recording of LSG activity}

The neural activity of the LSG was recorded in the sham-operated group and the AS group 3 days after the operation. The results are shown in Figure 4. Compared with those in the sham-operated group, the frequency and amplitude of the LSG were significantly elevated in the AS group (frequency: 254 \pm 34 versus $83 \pm 23$ impulses/min, $P<0.001$; amplitude: $0.0656 \pm 0.008$ versus $0.0377 \pm 0.004 \mathrm{mV}$, $P<0.001)$.
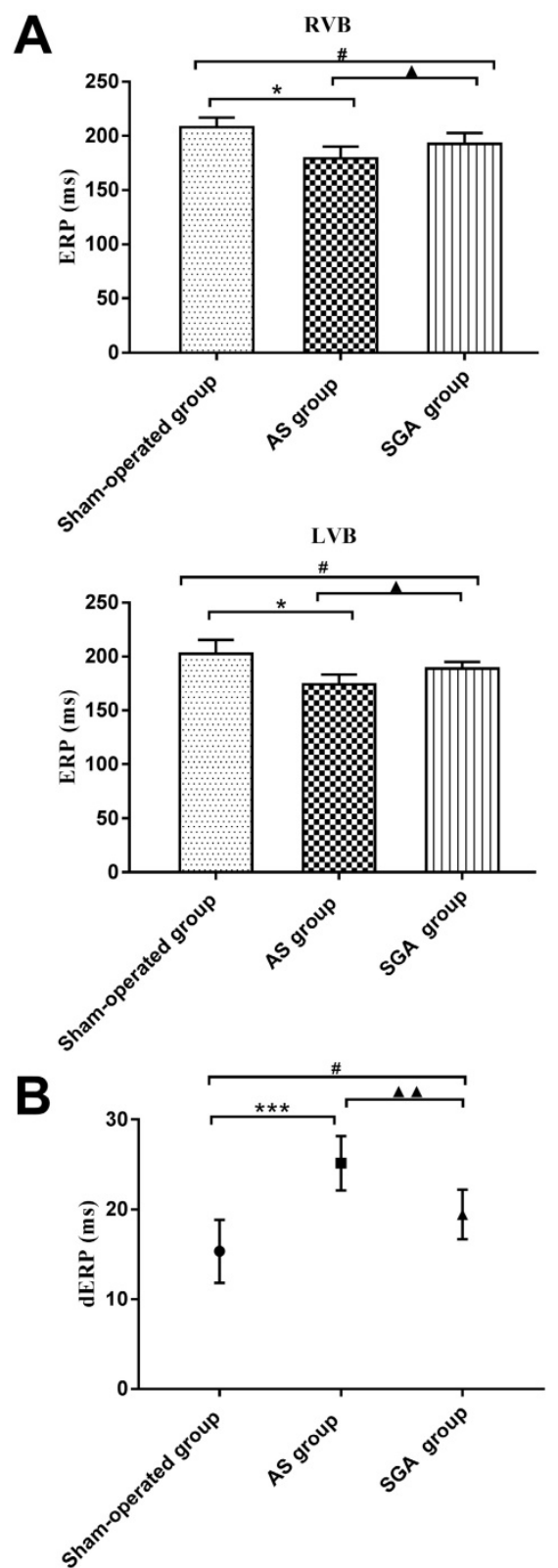
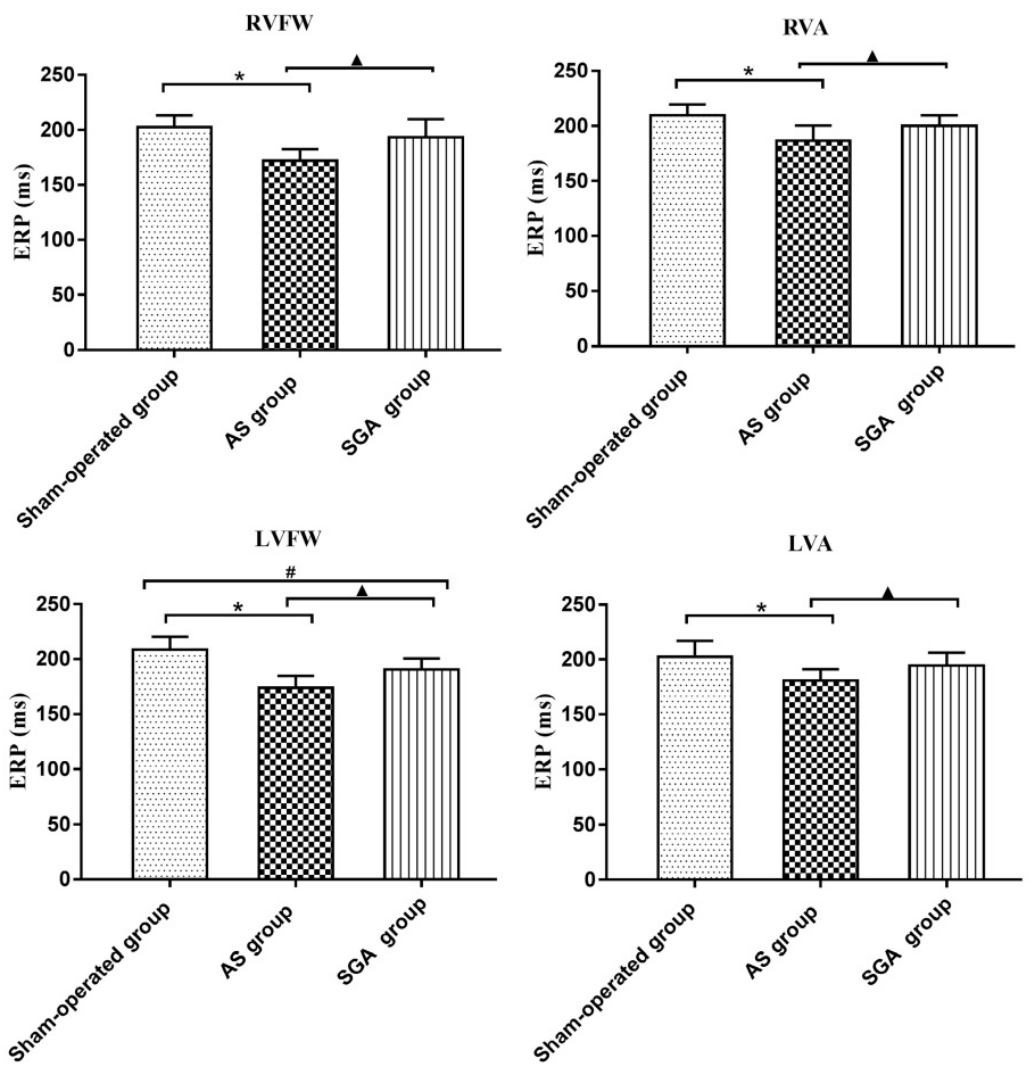

Figure 2: Differences in (A) the ventricular ERPs and (B) the dERP in all the groups. (A) The ERPs at all recording sites in the AS group were significantly reduced than those in the sham-operated group and the SGA group. The ERPs at RVB, LVB and LVFW were lower in the SGA group than those in the sham-operated group. However, there were no significant difference in ERPs at RVFW, RVA and LVA between the sham-operated group and the SGA group. ${ }^{* P}<0.05$; $\# P<0.05$; ${ }^{\wedge} P<0.05$. (B) The $d E R P$ in the AS group was significantly increased than that in the sham-operated group and the SGA group. The dERP was higher in the SGA group than that in the sham-operated group. $* * * P<0.001$; $\# P<0.05 ;{ }^{\Delta} \mathrm{P}<0.01$. Abbreviations: AS: acute stroke; SGA: stellate ganglion ablation; ERPs: effective refractory periods; dERP: dispersion of effective refractory period; RVA: right ventricular epicardium at the apex; RVFW: right ventricular epicardium at the free wall; RVB: right ventricular epicardium at the base; LVA: left ventricular epicardium at the apex; LVFW: left ventricular epicardium at the free wall; LVB: left ventricular epicardium at the base. 
A

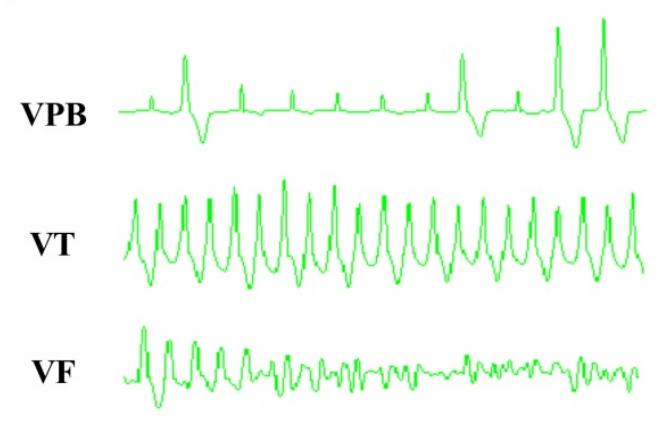

C

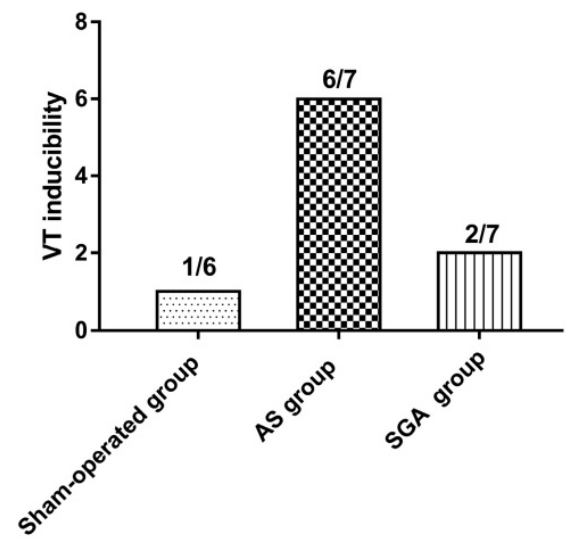

B

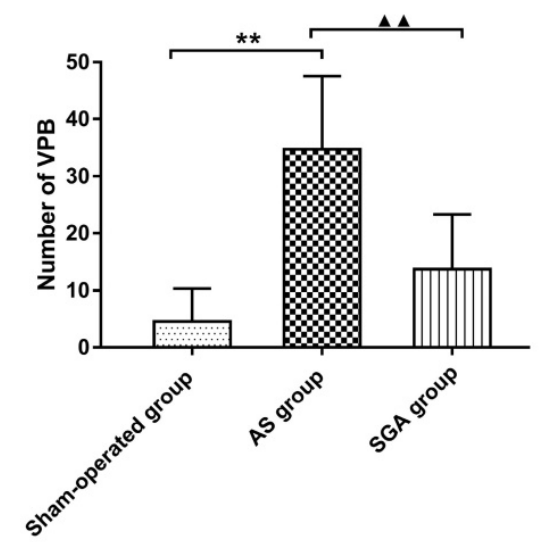

D

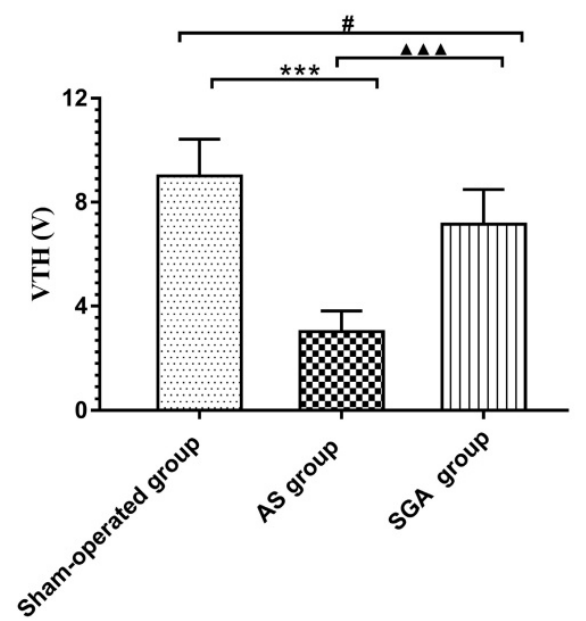

Figure 3: The number of (B) spontaneous VPB, (C) VT inducibility and (D) VFH in all the groups. (A) Examples of VPB, VT and VF occurrence. (B) The number of spontaneous VPB during the monitoring was increased than that in the sham-operated group and the SGA group. The SGA group had a higher number of VPB than the sham-operated group, but there was no statistical significant. ${ }^{* * P}<0.01 ;{ }^{\mathbf{\Lambda}} \mathrm{P}<0.01$. (C) VT was induced in 1 canine in the sham-operated group $(1 / 6), 6$ canines in the AS group $(6 / 7)$ and 2 canines in the SGA group (2/7). No VF was induced in all the groups. (D) The AS group had an significantly reduced VFH than the sham-operated group and the SGA group. Compared with that in the sham-operated group, the VFH in the SGA group was lower. ${ }^{* * * P}<0.001 ; \# P<0.05 ;{ }^{\Delta \Lambda}{ }^{\Delta}<<0.001$. Abbreviations: AS: acute stroke; SGA: stellate ganglion ablation; VPB: ventricular premature beat; VT: ventricular tachycardia; VF: ventricular fibrillation; VFH: ventricular fibrillation threshold.

\section{Measurements of NE levels in plasma and ventricle}

As shown in Table 1, the levels of NE in plasma and LV were significantly elevated in the AS group 3 days after MCAO. LSG ablation could suppress the elevated NE levels induced by acute stroke.

\section{Pro-inflammatory macrophages polarization in ventricle}

As shown in Figure $\mathbf{5 A}$ and $\mathbf{5 B}$, the number of pro-inflammatory M1 cells in ventricle was significantly increased in the AS group, compared with that in the sham-operated group (42 \pm 6 versus $14 \pm 4$ per $\left.\mathrm{mm}^{2}, \mathrm{P}<0.001\right)$ and the SGA group $(42 \pm 6$ versus $22 \pm 4$ per $\left.\mathrm{mm}^{2}, \mathrm{P}<0.001\right)$. The number of $\mathrm{M} 1$ cells in ventricle was higher in the SGA group than that in the sham-operated group $(\mathrm{P}<0.01)$. Real-time PCR of iNOS was also used for further clarification (Figure 5C). In the AS group, the relative levels of iNOS mRNA in ventricle were markedly elevated than those in the sham-operated group $(3.39 \pm 0.27$ versus $1.01 \pm 0.21, \mathrm{P}<0.001)$ and the SGA group $(3.39 \pm$ 0.27 versus $1.89 \pm 0.25, \mathrm{P}<0.001)$. The SGA group had higher levels of iNOS mRNA in ventricle compared with the sham-operated group $(\mathrm{P}<0.001)$.

\section{Levels of inflammatory cytokines in ventricle}

All immunoblot band intensity measurements were normalized to the intensity of the GAPDH band in the loaded sample. As shown in Figure 6, the levels of MCP-1 and TNF-a in ventricle were markedly increased in the AS group, compared with those in the sham-operated group (TNF-a: $0.57 \pm 0.06$ versus 0.14 \pm 0.04; MCP-1: $0.54 \pm 0.05$ versus $0.15 \pm 0.05 ; \mathrm{P}<0.001$ ) and the SGA group (TNF-a: $0.57 \pm 0.06$ versus $0.28 \pm$ 0.06; MCP-1: $0.54 \pm 0.05$ versus $0.32 \pm 0.05 ; \mathrm{P}<0.001$ ). The levels of MCP-1 and TNF-a in ventricle were higher in the SGA group than those in the sham-operated group $(\mathrm{P}<0.01)$. As shown in Figure 7, the expression of NF- $\mathrm{KB}$ p65 protein in ventricle was 
markedly higher in the AS group, compared with that in the sham-operated group $(0.59 \pm 0.07$ versus $0.13 \pm$ $0.04, \mathrm{P}<0.001)$ and the SGA group $(0.59 \pm 0.07$ versus $0.22 \pm 0.06, \mathrm{P}<0.001)$. The expression of NF-kB p65 in ventricle was higher in the SGA group than that in the sham-operated group $(\mathrm{P}<0.01)$.

\section{Discussion}

This study investigated the influence of SGA on VAs in a canine model of MCAO and its potential mechanism. We provided evidence of the following: (1) An acute ischemic stroke can lead to the increased risk of VAs, which may be associated with the pro-inflammatory polarization of macrophages induced by sympathetic excessive activity; and (2) LSG ablation can inhibit the occurrence of VAs after an acute stroke by downregulating the catecholamine levels in plasma and preventing macrophages activating in myocardium.

A

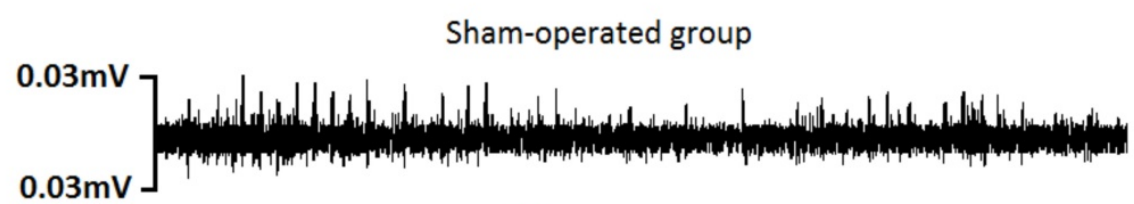

AS group

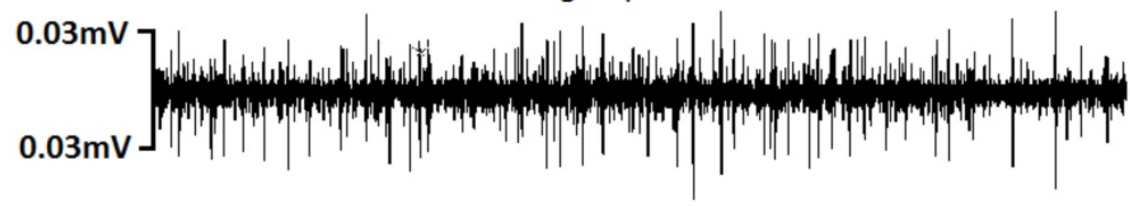

B

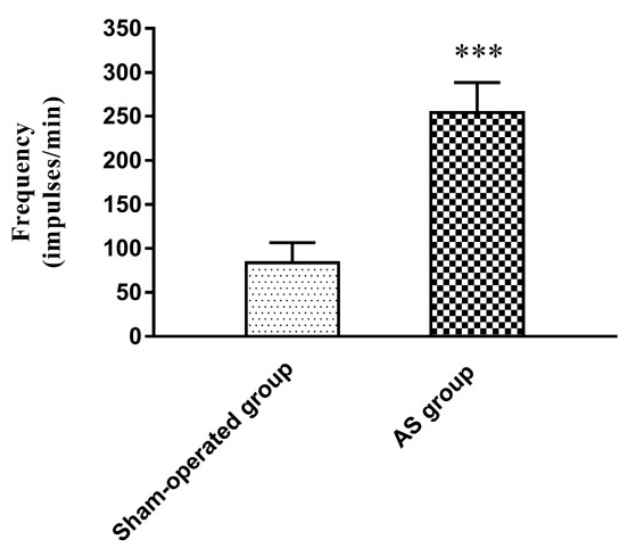

C

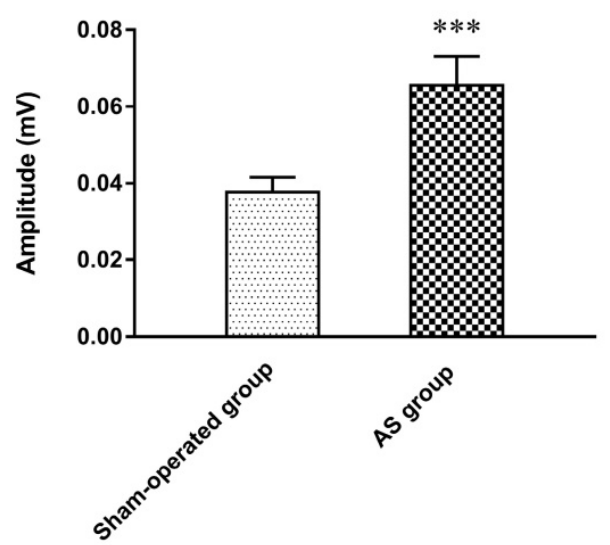

Figure 4: (A) Examples of LSG activity 3 days after operation. (B) The frequency and (C) the amplitude in the AS group were greatly elevated compared with those in the sham-operated group. ${ }^{*} * * \mathrm{P}<0.001$. Abbreviations: LSG: left stellate ganglion; AS: acute stroke.

Table 1. Difference of NE levels in plasma and LV

\begin{tabular}{llllll}
\hline & \multicolumn{2}{l}{ Sham-operated group } & AS group & & SGA group \\
\hline NE $(\mathrm{pg} / \mathrm{ml})$ & base & 3 days later & base & 3 days later & base \\
plasma & $431.7 \pm 53.8$ & $451.1 \pm 43.5$ & $436.2 \pm 46.4$ & $692.8 \pm 44.4^{* * *}$ & $419.4 \pm 45.2$ \\
LV & & $139.9 \pm 21.7$ & & $351.8 \pm 30.3^{* * *}$ & $542.1 \pm 50.1^{\# \# \# \boldsymbol{\Lambda}}$ \\
\hline
\end{tabular}

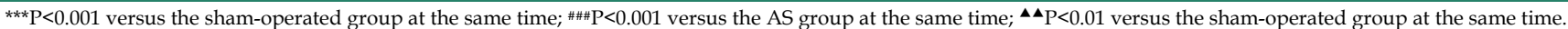
Abbreviations: AS: acute stroke; SGA: stellate ganglion ablation; NE: norepinephrine; LV: left ventricle. 

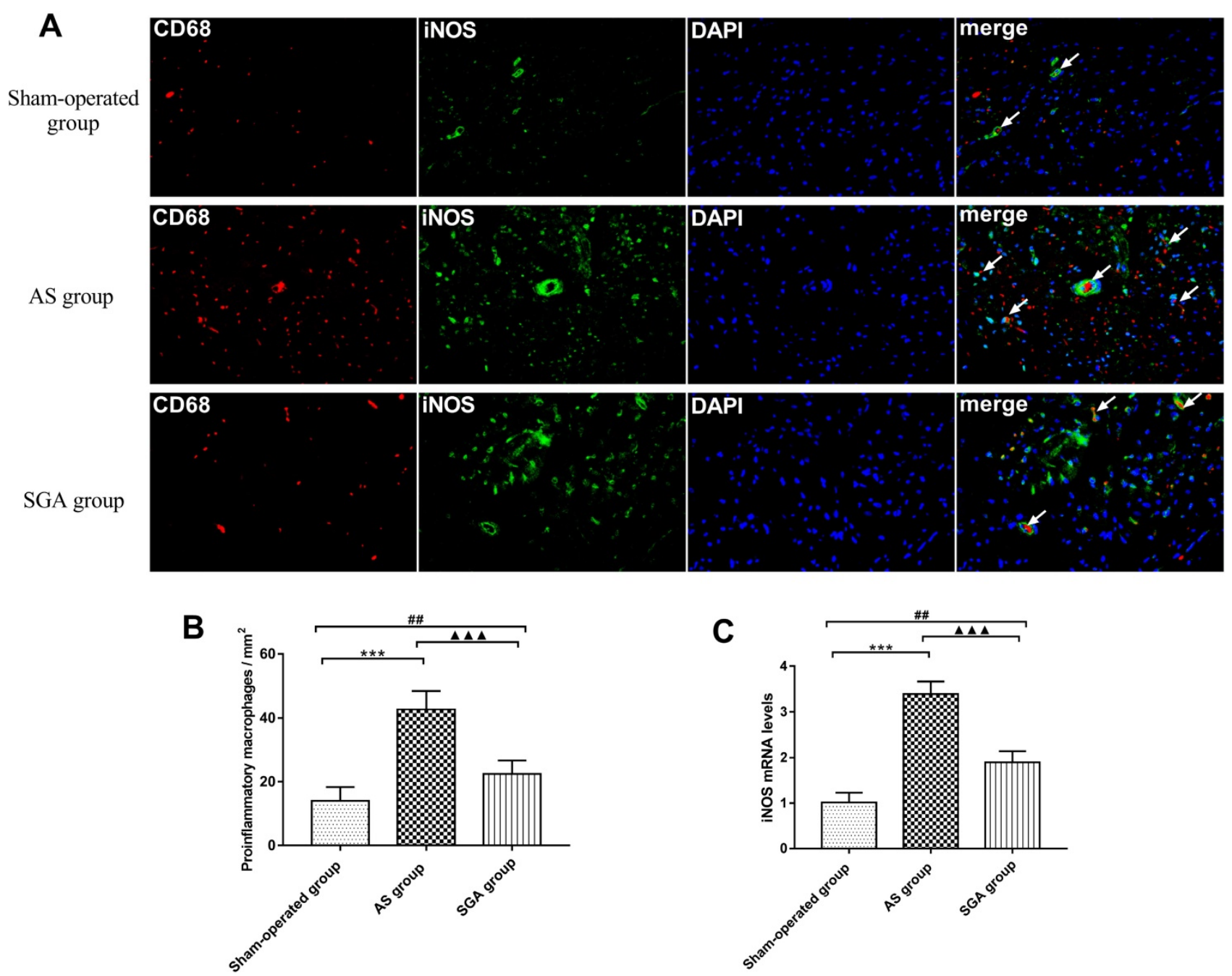

Figure 5: (A) The immunofluorescence of pro-inflammatory $M 1$ macrophages in ventricle. (B) the number of pro-inflammatory $M 1$ macrophages in ventricle was significantly increased in the AS group than that in the sham-operated group and the SGA group. The SGA group had a higher number of MI macrophages in ventricle compared with the sham-operated group. (C) The relative levels of iNOS mRNA in ventricle were significantly increased in the AS group than those in the sham-operated group and the SGA group. The SGA group had higher levels of iNOS mRNA in ventricle compared with the sham-operated group. ${ }^{* * *} \mathrm{P}<0.001 ;{ }^{*} \mathrm{P}<0.01 ;{ }^{\mathbf{\Lambda} \wedge}{ }^{\mathbf{A}} \mathrm{P}<0.001$. Abbreviations: AS: acute stroke; SGA: stellate ganglion ablation; iNOS: inducible nitric oxide synthase.

Abnormal ECGs and cardiac arrhythmias are commonly identified in patients with acute stroke, varying from abnormal $\mathrm{T}$ waves, to QT prolongation, to fatal arrhythmias, such as VF that causes sudden cardiac death [13]. The previous clinical studies have suggested that, acute strokes, especially involving the insular cortex, increase the incidence of VAs through a cascade of events that alter autonomic balance and increase catecholamine levels. Besides VAs, the typical myocardial damage induced by acute stroke has also been commonly observed [14, 15]. The interval between the stroke onset and the consequence of ventricular arrhythmogenesis has not been clarified because the long-term studies with large sample sizes are lacking. According to the available studies, new VAs are mostly detected within 3 days after the stroke onset [16, 17]. In the present study, although we failed to perform the postoperative continuous ECG monitoring, we found that the number of spontaneous VPB was markedly increased in the AS group during the 1 hour ECG monitoring. Besides, the AS group showed significantly reduced ERPs and TFV, increased dERP and VT inducibility at programmed electrical stimuli 3 days after the MCAO.

Clinical and experimental researches have shown that autonomic dysfunction is commonly observed after an acute stroke, mainly manifested as sympathetic overactivation $[5,6]$. The sympathetic excessive activity and sympathetic nerve sprouting in myocardium have been demonstrated to play important roles in VAs vulnerability. By contrast, the decreased sympathetic nerve activity such as renal denervation or LSG inhibition may have assisted in suppressing VAs. Anatomically, the majority of cardiac sympathetic axons and nerve terminals originate in the cell bodies of the SG. It is well known that the LSG highly involves in the regulation of 
ventricular electrophysiology. Yu et al. [18] found that LSG inhibition could stabilize cardiac electrophysiology and suppress susceptibility to ischemia induced VAs in canines. Vaseghi et al. [19] demonstrated that LSG denervation could effectively decrease sustained VT and implantable cardioverter-defibrillator shock recurrence in patients with refractory VT. In the present study, we found that neural activity of the LSG significantly increased, combined with elevated serum levels of NE in canines with acute stroke. LSG ablation could significantly attenuate the shortened ERPs and the increased VAs vulnerability caused by acute ischemic stroke.

The previous studies have demonstrated that inflammatory responses are involved in the pathogenesis of VAs induced by myocardial infarction via degradation connexin 43 (CX43) [20], and the enhanced inflammatory responses are associated with LSG remodeling through sympathetic nerve activation [21]. Furthermore, macrophages activation as well as pro-inflammatory M1 phenotype polarization plays a crucial role in mediating infarction-related inflammatory responses and electrical conduction in the heart [22, 23]. A recent histopathologic study documented that the infiltration of macrophages was much higher in the myocardium of patients who died after subarachnoid hemorrhage [24]. However, the association between the cardiac macrophages activation and the acute ischemic stroke has not been well explored. In the present study, we found that the infiltration of M1 macrophages was increased, combined with the elevated levels of MCP-1 and TNF- $\alpha$ in ventricle in canines with acute ischemic stroke, which could be attenuated by LSG ablation.
A

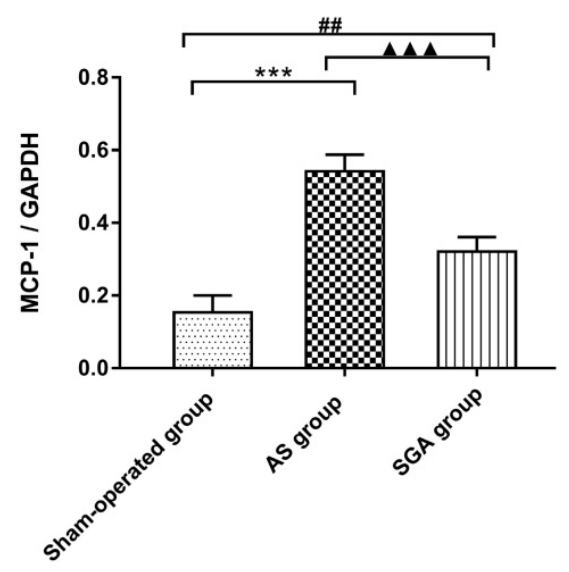

C

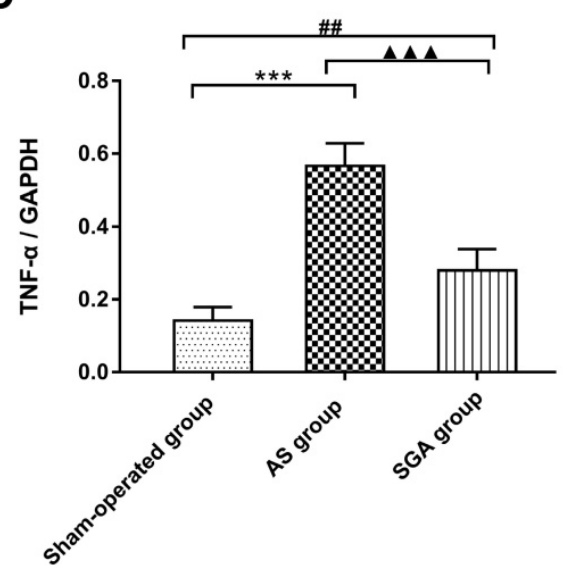

B

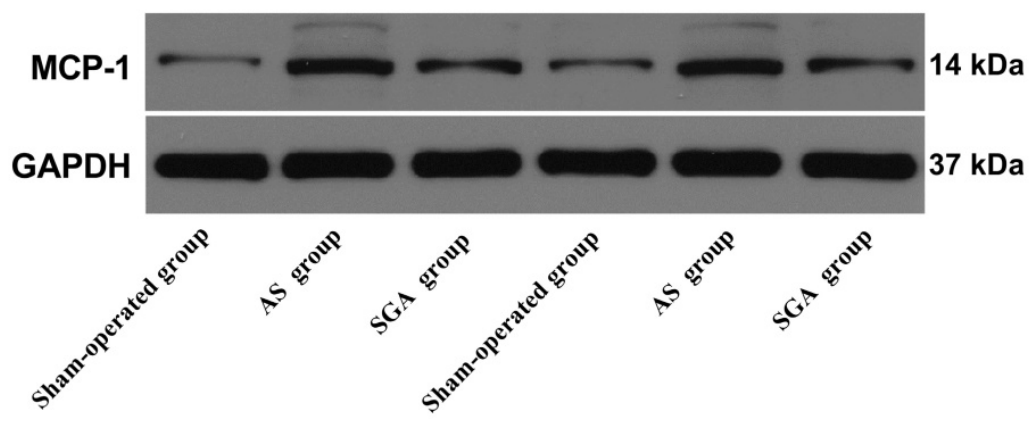

D

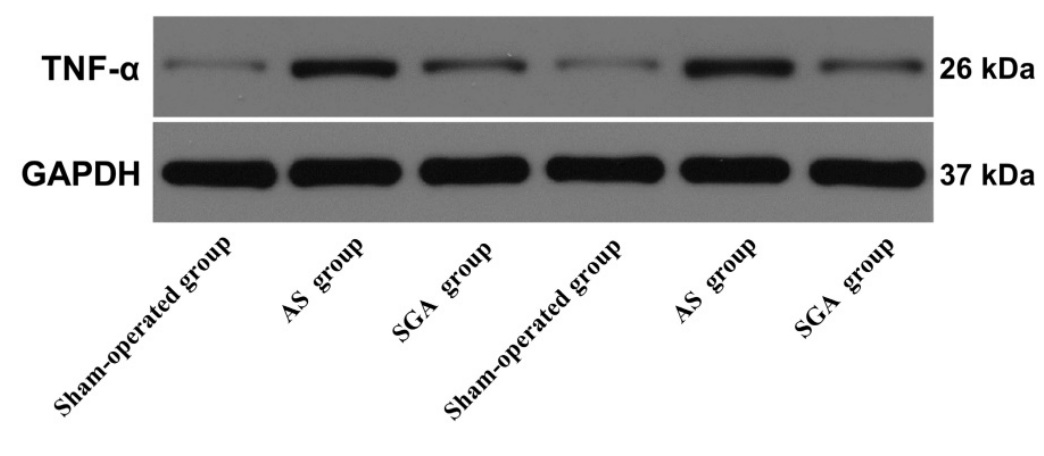

Figure 6: (A) The levels of MCP-1 and (D) TNF- $\alpha$ in ventricle were significantly increased in the AS group, compared with those in the sham-operated group and the SGA group. The SGA group had higher levels of MCP-1 and TNF- $\alpha$ in ventricle than the sham-operated group. ${ }^{* * *} \mathrm{P}<0.001 ;{ }^{\# P} \mathrm{P}<0.01 ;{ }^{\mathbf{\Lambda}}{ }^{\mathbf{\Lambda}} \mathrm{P}<0.001$. (B) and (D) The Western blotting results of MCP-1 and TNF- $\alpha$ in ventricle. Abbreviations: AS: acute stroke; SGA: stellate ganglion ablation; MCP-1: monocyte chemotactic protein-1; TNF- $\alpha$ : tumour necrosis factor-alpha. 
A

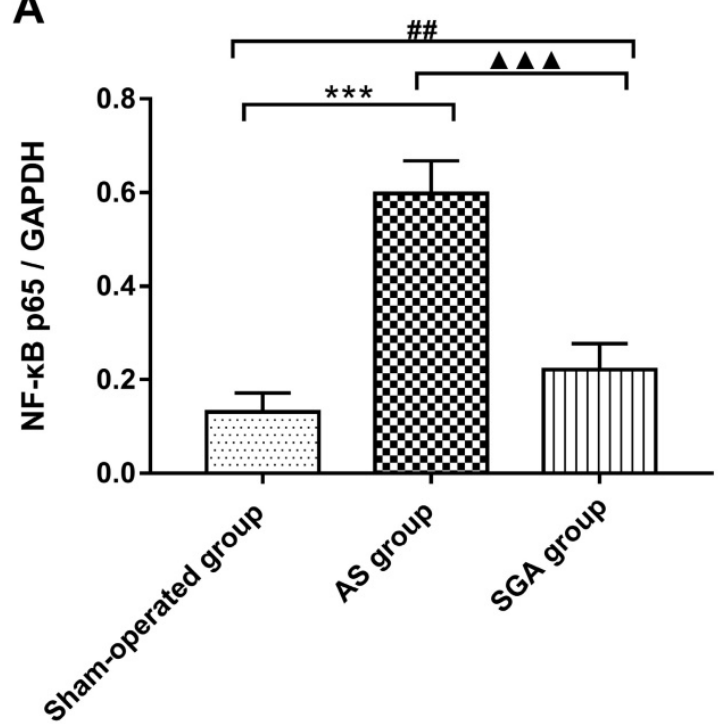

B

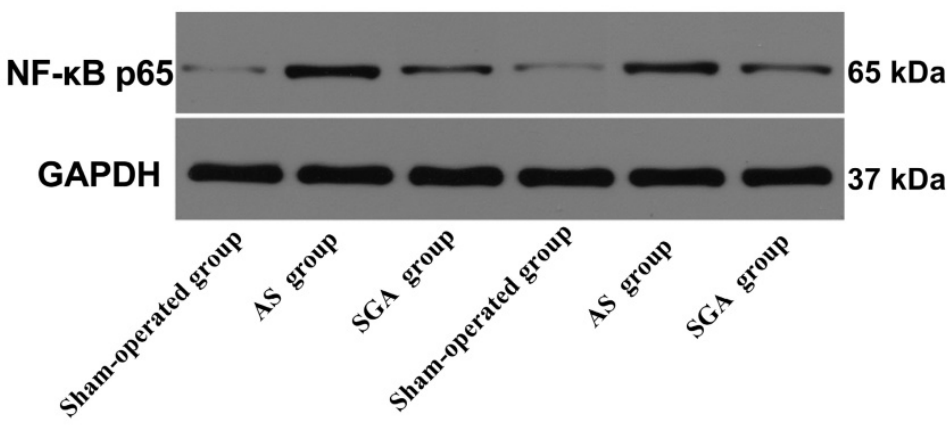

Figure 7: (A) The expression of NF-KB P65 protein in ventricle was significantly elevated in the AS group, compared with that in the sham-operated group and the SGA group. The SGA group had a higher expression of NF-KB P65 protein in ventricle than the sham-operated group. ${ }^{* * *} \mathrm{P}<0.001 ;{ }^{\# P} \mathrm{P}<0.01 ;{ }^{\mathbf{\Lambda}}{ }^{\mathbf{4}} \mathrm{P}<0.001$. (B) The Western blotting results of NF-KB P65 protein in ventricle. Abbreviations: AS: acute stroke; SGA: stellate ganglion ablation.

It has been demonstrated that NE is a potential pro-inflammatory mediator and can induce the secretion of inflammatory cytokines via macrophages activation [8]. Li et al. [9] have demonstrated that NE is capable of inducing IL-6 production in macrophages via the $\beta$-adrenoreceptor-NAD $(\mathrm{P}) \mathrm{H}$ oxidase system-NF-kB signaling pathway. The NF-kB signaling pathway promotes pro-inflammatory M1 polarization and plays an essential role in macrophage-initiated inflammatory responses [25]. In the present study, we found that the level of NF-kB p65 in ventricle elevated after acute ischemic stroke and that LSG ablation could reduce NF-kB p65 expression. These results suggested that sympathetic excessive activity after acute stroke induced M1 macrophages polarization and inflammatory chemokines secretion possibly via the NF-kB signaling pathway.

\section{Conclusions}

In our study, we demonstrated that sympathetic hyperactivity caused by acute stroke could lead to the increased VAs vulnerability through the macrophages polarization and the secretion of inflammatory cytokines. LSG ablation could suppress the activity of macrophages and thereby inhibit VAs occurrence after acute stroke.

\section{Study limitations}

This study has several limitations. Firstly, in this study, we failed to conduct postoperative continuous ECG monitoring to record the spontaneous VAs after acute stroke. Secondly, we did not measure the action potential duration (APD) and monophasic action potential (MAP) in endocardium and epicardium. Thirdly, we failed to investigate the effects of bilateral SGA on stroke induced VAs for comparison. Finally, previous studies have suggested the asymmetry of the bilateral insular cortex in the regulation of the autonomic nervous system. We did not explore the effects of different brain lesion locations on VAs. Whether damages of different areas have different influences on VAs remains unknown.

\section{Acknowledgements}

The authors are grateful for the kind support from Yanhong Tang, Xi Wang and Teng Wang (Hubei Key Laboratory of Cardiology, Wuhan, China).

\section{Funding}

This work was supported by the National Natural Science Foundation of China (No.81670303 and 81970277 to QY Zhao).

\section{Competing Interests}

The authors have declared that no competing interest exists.

\section{References}

1. Norris JW, Froggatt GM, Hachinski VC. Cardiac arrhythmias in acute stroke. Stroke. 1978. 9(4): 392-6.

2. Koppikar S, Baranchuk A, Guzmán JC, et al. Stroke and ventricular arrhythmias. Int J Cardiol. 2013. 168(2): 653-9.

3. Sörös P, Hachinski V. Cardiovascular and neurological causes of sudden death after ischaemic stroke. Lancet Neurol. 2012. 11(2): 179-88.

4. Chidambaram H, Gnanamoorthy K, Suthakaran PK, et al. Assessment of Autonomic Dysfunction in Acute Stroke Patients at a Tertiary Care Hospital. J Clin Diagn Res. 2017. 11(2): OC28-OC31.

5. Back C, Thiesen KL, Skovgaard K, et al. RAAS and stress markers in acute ischemic stroke: preliminary findings. Acta Neurol Scand. 2015. 131(2): 132-9.

6. Xiong L, Leung HH, Chen XY, et al. Comprehensive assessment for autonomic dysfunction in different phases after ischemic stroke. Int J Stroke 2013;8:645-651. 
7. Li CY, Li YG. Cardiac Sympathetic Nerve Sprouting and Susceptibility to Ventricular Arrhythmias after Myocardial Infarction. Cardiol Res Pract. 2015. 2015: 698368.

8. Huang JL, Zhang YL, Wang CC, et al. Enhanced phosphorylation of MAPKs by NE promotes TNF- $\alpha$ production by macrophage through a adrenergic receptor. Inflammation. 2012. 35(2): 527-34.

9. $\mathrm{Li} \mathrm{M}$, Yao $\mathrm{W}$, $\mathrm{Li} \mathrm{S}$, et al. Norepinephrine induces the expression of interleukin-6 via $\beta$-adrenoreceptor- $\mathrm{NAD}(\mathrm{P}) \mathrm{H}$ oxidase system-NF- $\mathrm{kB}$ dependent signal pathway in U937 macrophages. Biochem Biophys Res Commun. 2015. 460(4): 1029-34.

10. Zhang WH, Zhou QN, Lu YM, et al. Renal Denervation Reduced Ventricular Arrhythmia After Myocardial Infarction by Inhibiting Sympathetic Activity and Remodeling. J Am Heart Assoc. 2018. 7(20): e009938.

11. Liu S, Yu X, Luo D, et al. Ablation of the Ligament of Marshall and Left Stellate Ganglion Similarly Reduces Ventricular Arrhythmias During Acute Myocardial Infarction. Circ Arrhythm Electrophysiol. 2018. 11(5): e005945.

12. Wang $\mathrm{Y}$, Xiong $\mathrm{X}, \mathrm{Xie} \mathrm{B}$, et al. A brain-stellate ganglion-atrium network regulates atrial fibrillation vulnerability through macrophages in acute stroke. Life Sci. 2019. 237: 116949.

13. Wang Y, Qian Y, Smerin D, et al. Newly Detected Atrial Fibrillation after Acute Stroke: A Narrative Review of Causes and Implications. Cardiology. 2019. 144(3-4): 112-121.

14. Simula S, Muuronen AT, Taina M, et al. Effect of middle cerebral artery territory ischemic stroke on QT interval. J Stroke Cerebrovasc Dis. 2014. 23(4): 717-23.

15. Baranchuk A, Nault MA, Morillo CA. The central nervous system and sudden cardiac death: what should we know. Cardiol J. 2009. 16(2): 105-12.

16. Ruthirago D, Julayanont $\mathrm{P}$, Tantrachoti $\mathrm{P}$, et al. Cardiac Arrhythmias and Abnormal Electrocardiograms After Acute Stroke. Am J Med Sci. 2016. 351(1): 112-8.

17. Orlandi G, Fanucchi S, Strata G, et al. Transient autonomic nervous system dysfunction during hyperacute stroke. Acta Neurol Scand. 2000. 102(5): 317-21.

18. Yu L, Wang M, Hu D, et al. Blocking the Nav1.8 channel in the left stellate ganglion suppresses ventricular arrhythmia induced by acute ischemia in a canine model. Sci Rep. 2017. 7(1): 534.

19. Vaseghi M, Barwad P, Malavassi CFJ, et al. Cardiac Sympathetic Denervation for Refractory Ventricular Arrhythmias. J Am Coll Cardiol. 2017. 69(25): 3070-3080.

20. De Jesus NM, Wang L, Herren AW, et al. Atherosclerosis exacerbates arrhythmia following myocardial infarction: Role of myocardial inflammation. Heart Rhythm. 2015. 12(1): 169-78.

21. Yu L, Wang $Y, Z$ Zhou $X$, et al. Leptin injection into the left stellate ganglion augments ischemia-related ventricular arrhythmias via sympathetic nerve activation. Heart Rhythm. 2018. 15(4): 597-606.

22. Lambert JM, Lopez EF, Lindsey ML. Macrophage roles following myocardial infarction. Int J Cardiol. 2008. 130(2): 147-58

23. Hulsmans M, Clauss $S$, Xiao L, et al. Macrophages Facilitate Electrical Conduction in the Heart. Cell. 2017. 169(3): 510-522.e20.

24. van der Bilt IA, Vendeville JP, van de Hoef TP, et al. Myocarditis in patients with subarachnoid hemorrhage: A histopathologic study. J Crit Care. 2016. 32: 196-200.

25. Lowe JM, Menendez D, Bushel PR, et al. p53 and NF-kB coregulate proinflammatory gene responses in human macrophages. Cancer Res. 2014. 74(8): 2182-92 